\title{
Transport Coefficients in Moderately Dense Gases
}

\author{
Siegrried Grossmann \\ Institut für Theoretische Physik der Universität Marburg \\ (Z. Naturforschg. 20 a, 861-869 [1965] ; received 10 April 1965)
}

\begin{abstract}
The first virial corrections for shear viscosity $\eta$ and thermal conductivity $\lambda$ are calculated, starting from the BoltzManN-Landau equation of a quantum gas in binary collision approximation. The first order Chapman-Enskog solution is used. With a very simplified collisional operator numerical values are got and compared with experiments. The result is that the kinetic equation including the interaction effects in quasi-free motion is able to explain the empirical order of magnitude of $\mathrm{d} \eta / \mathrm{d} n$.
\end{abstract}

Recently attempts have been made, to calculate transport coefficients of gases for not too low densities. A summary has been given by Cohen, Ernst, and Dorfmann ${ }^{1}$, RÉsibors ${ }^{2}$ and Kawasaki and OpPENHEIM ${ }^{3}$.

There are three methods:

(i) Te earliest was that of ENskog ${ }^{4}$, starting from a modified Boltzmann equation for hard cores.

(ii) Another one starts from a formula, which expresses the transport coefficient by time-correlation functions ${ }^{5}$.

Or: (iii) The transport properties are given by certain integrals over reduced non-equilibrium distribution functions ${ }^{6}$. The equivalence of the last methods has been shown recently by RÉsiBors ${ }^{2}$ for classical gases. (See also ${ }^{3}$.)

In this paper we will return to a Boltzmann equation approach to transport theory. This has been very successful for low density gases. Of course, the wellknown Boltzmann equation is not capable of treating gases at moderate densities: it leads to equilibrium properties of perfect gases and to lowest-order-in-density transport coefficients. To extend the range of application with respect to the density, Boltzmans's equation for the particle distribution function $f(\boldsymbol{r}, \boldsymbol{p}, t)$ must be extended first. One attempt in doing that is EnsкoG's proposal for a modified collisional change $\partial_{c} f / \partial t$. Its main ad-

1 E. G. D. Cohen and M. H. J. J. Ernst, Phys. Letters 5, 192 [1963] ; 12, 319 [1964].

2 P. Rsibois, J. Chem. Phys. 41, 2979 [1964].

3 K. Kawasaki and J. Oppenheim, Phys. Rev. 136, A 1519 [1964].

4 See e.g. S. Chapman and T. G. Cowling, The Mathematical Theory of Non Uniform Gases, University Press, Cambridge 1953.

5 See e. g. J. A. McLennan, Advan. Chem. Phys. 5, 261 [1963] or P.C. Martin, in Statistical Mechanics of Equilibrium vantage is that the transport coefficients can be calculated up to very high densities even near the critical region. Its disadvantages are that it can only be formulated for a hard sphere gas, and that an ad hoc modification of the final results (together with an additional fitting of an interaction parameter) is necessary to get correct results. Numerically they agree with experiments over a large range of density, but at small densities there are considerable deviations between the ENskog theory and experiment ${ }^{7}$. Now recently methods have been developed to generalize transport equations to systems with arbitrary densities, based on ideas of Bogoliubov, Landau, Kirkwood, Cohen, Prigogine etc. For quantum gases especially in the binary collision approximation it has been shown ${ }^{8}$ that a simply altered Boltzmann equation of the LANDAU-type very well describes moderately dense gases with arbitrary (but short range, of course) interaction. The point is: the influence of the interaction on a given molecule is not only described by collisions, between which the particles are free - there is, moreover, a mean interaction also during the time of mean free flight $\tau$, modifying the energy $\varepsilon_{p}$ of molecule with momentum $p$.

$$
\begin{gathered}
\varepsilon_{p}(r, t)=\frac{p^{2}}{2 m}+\int F(\boldsymbol{p}, \boldsymbol{q}) f(\boldsymbol{r}, \boldsymbol{q}, t) \mathrm{d} \boldsymbol{q} . \\
F(\boldsymbol{p}, \boldsymbol{q})=F(\boldsymbol{q}, \boldsymbol{p})=F(k) ; \quad \boldsymbol{k}=\frac{1}{2}(\boldsymbol{p}-\boldsymbol{q})
\end{gathered}
$$

and Non-Equilibrium, Ed. J. Merxner, North-Holland Publ. Comp., Amsterdam 1965.

6 See e. g. E. G. D. Cohen, Fundamental Problems in Statistical Mechanics, North-Holland Publishing Co., Amsterdam 1962, p. 110.

7 American Institut of Physics Handbook, 1964, Chapters $2-223$ and $4-80$.

8 S. Grossmann, Z. Phys. 182, 24 [1964] ; Nuovo Cim. 37, 698 [1965]. 
is the real part of the two particle scattering amplitude in forward direction ${ }^{8}$. If we restrict to binary collision approximation, it is independent of $f$. The collisional operator in the transport equation is altered in containing the conservation law for the energy (1), besides momentum conservation as usual. As $\varepsilon_{p}$ depends on the distribution $f$, the collisional operator $\sigma$ gets density dependent.

According to the idea of LANDAU ${ }^{9}$ also the streaming part of the transport equation has to be altered. The velocity of a quantum particle with dispersion relation (1) is

$$
\frac{\partial \varepsilon_{p}}{\partial \boldsymbol{p}}=\frac{\boldsymbol{p}}{m}+\frac{\partial}{\partial \boldsymbol{p}} \int F(\boldsymbol{p}, \boldsymbol{q}) f(\boldsymbol{r}, \boldsymbol{q}, t) \mathrm{d} \boldsymbol{q}
$$

and its acceleration is

$-\frac{\partial \varepsilon_{p}}{\partial \boldsymbol{r}}=\int F(\boldsymbol{p}, \boldsymbol{q}) \frac{\partial f(\boldsymbol{r}, \boldsymbol{q}, t)}{\partial \boldsymbol{r}} \mathrm{d} \boldsymbol{q} \sim$ density .

The distribution function thus must be a solution of

$$
\begin{aligned}
\frac{\partial f}{\partial t} & +\frac{\partial \varepsilon_{p}}{\partial \boldsymbol{p}} \cdot \frac{\partial f}{\partial \boldsymbol{r}}-\frac{\partial \varepsilon_{p}}{\partial \boldsymbol{r}} \cdot \frac{\partial f}{\partial \boldsymbol{p}} \\
& =\int \sigma\left(p, p_{1} \rightarrow p^{\prime} p_{1}^{\prime} ; f\right)\left(f^{\prime} f_{1}^{\prime}-f f_{1}\right) \mathrm{d} p_{1} \mathrm{~d} p^{\prime} \mathrm{d} p_{1}{ }^{\prime} .
\end{aligned}
$$

Statistics effects have been neglected. As is shown in ${ }^{8}$, this Boltzmann-Landau equation leads to quasi-equilibrium properties of a real gas: virial equation of state, density-corrections for local energy, entropy etc., a modification of the sound velocity $c=c(n)$ and the prediction of a physical phenomenon in gases, which is known from low temperature quantum liquids: Zero sound is likely to be expected in a moderately dense Boltzmann gas for that region of temperature, in which the repulsive component of the intermolecular force predominates. All these quasi-equilibrium effects are only due to the modified streaming part of (5), because the r.h.s. does not appear in the conservation laws, which govern quasi-equilibrium behaviour. This shows the importance of the modifications (3) and (4). Its lack in the ENskog theory would lead to wrong results for the transport coefficients, if it were not added indirectly as so-called "collisional transfer" of momentum etc. This has only be formulated for hard spheres. The BoLtzMANN-LANDAU equation (5) thus seems to be a generalization of ENsкoG's theory of moderately dense gases with arbitrary interaction, provided a transport equation of MARKоFF-type is correct at all.

9 L. D. Landau, Soviet Phys.-JETP 3, 920 [1957] ; 5, 101 [1957].
The modified streaming part is also very plausible from the derivation of the transport equation from first principles. The $S$-matrix in the collisional change only occurs if one integrates the Liouville or von Neumans equation over a finite time $t \ldots$ $t+\Delta t$. This must necessarely change the pure motion, which only infinitesimally is described by $\boldsymbol{v} \cdot \operatorname{grad}_{\boldsymbol{r}}$. In finite time intervals "interference" between $H_{\text {kin }}$ and $V$ occurs, altering the streaming part simultaneously with the collisional part. In fact both are mixed and it is somewhat artificially to separate them into a term, in which momentum change predominates $\left(\partial_{c} f / \partial t\right)$ and another, in which position change predominates. But as long as the system is gas-like, this picture should be true, at least approximatively.

In this paper we will study the transport coefficients for shear viscosity $(\eta)$ and heat conduction ( $\lambda$ ) based on (5). As is well known, this requires (even in the low density limit) the solution of an integral equation with the collisional operator as kernel. It has been shown by RÉsIBoIs ${ }^{2}$ very clearly that all other methods of calculating transport coefficients also meet the very same integral equation for the linear deviation of the distribution function from (local) equilibrium. We will not check here the principal equivalence of our approach to the others. It clearly reduces to the well-known results for very dilute gases if $n \approx 0$. At moderate densities there are density corrections which could be calculated e. g. with perturbation methods. But in order to get a first test for the quality of (5) with respect to the density dependence of $\eta$ and $\lambda$, we will not write down the whole generalized theory of integration of (5), but will proceed by simplifying the collisional operator:

$$
\partial_{c} f / \partial t \approx-\left(f-f_{0}\right) / \tau \text {. }
$$

It is well known for very dilute gases (see e. g. ${ }^{10}$ ) that some typical features of transport theory may already be got by using such simplified collisional operator.

The accurate integration theory would lead to convolution integrals of $F(p, q)$ with $\sigma$, adding some more numerical trouble to the usual calculations of $\eta$ and $\lambda$. This cannot be done unless $F$ is known, either by calculating the real part of the

10 K. Huang, Statistical Mechanics, John Wiley \& Sons, Inc., New York - London 1963. 
scattering amplitude or by inverting

$$
B(T)=\left\langle\frac{\beta}{2} F(p, q)\right\rangle_{0}=\left\langle\frac{\beta}{2} F(k)\right\rangle_{0},
$$

which connects the second virial coefficient $B$ with $F^{8}$. As long as such results are not available one would try to factorize the convolutions, which essentially means to neglect the dependence of $F$ and $\sigma$ from its variables. This in turn allows the simplification (6).

\section{Approximate Solution of the Boltzmann-Landau Equation}

If the collisions have a very strong effect, almost immediately local equilibrium is established. $f$ is fully determined by local density, mean velocity and temperature:

$$
\begin{aligned}
f_{0}(\boldsymbol{r}, \boldsymbol{p}, t) & =\exp \left\{\beta\left(\alpha+\boldsymbol{p} \cdot \boldsymbol{u}-\varepsilon_{p}\left(f_{0}\right)\right)\right\}, \\
n(\boldsymbol{r}, t) & =\int f_{0}(\boldsymbol{r}, \boldsymbol{p}, \mathrm{t}) \mathrm{d} \boldsymbol{p}, \\
\boldsymbol{u}(\boldsymbol{r}, t) & =\left\langle\frac{\boldsymbol{p}}{m}\right\rangle_{f_{0}}=\left\langle\frac{\partial \varepsilon_{p}\left(f_{0}\right)}{\partial \boldsymbol{p}}\right\rangle_{f_{0}} .
\end{aligned}
$$

Particle, momentum and energy transport in such gases only occurs by convection. We are now interested in small deviations from quasi-equilibrium.

$$
f=f_{0}+f_{1} \text { and } f_{1} \ll f_{0} .
$$

Formally one takes care of deviations $\xi$ from local equilibrium $\xi=0$ by a factor $1 / \xi$ before $\partial_{c} t / \partial t$. Putting

$$
\begin{gathered}
f=f_{0}+\xi f_{1}+\xi^{2} f_{2}+\ldots \\
\text { we get }\left.\quad \frac{\partial f_{0}}{\partial t}\right|_{\xi=0}=0 \rightarrow(8) . \\
\frac{\partial f_{0}}{\partial t}+\frac{\partial \varepsilon_{p}\left(f_{0}\right)}{\partial \boldsymbol{p}} \cdot \frac{\partial f_{0}}{\partial \boldsymbol{r}}-\frac{\partial \varepsilon_{p}\left(f_{0}\right)}{\partial \boldsymbol{r}} \cdot \frac{\partial f_{0}}{\partial \boldsymbol{p}}=L\left(f_{1}\right) .
\end{gathered}
$$

The linear operator $L$ is given by

$$
\begin{aligned}
& L\left(f_{1}\right)=\int\left[\sigma ( f _ { 0 } ) \left(f_{0}{ }^{\left(1^{\prime}\right)} f_{1}{ }^{\left(2^{\prime}\right)}+f_{0}{ }^{\left(2^{\prime}\right)} f_{1}{ }^{\left(1^{\prime}\right)}\right.\right. \\
&\left.-f_{0}{ }^{(p)} f_{1}^{(1)}-f_{0}^{(1)} f_{1}{ }^{(p)}\right) \\
&\left.+\frac{\delta \sigma}{\delta f} \cdot f_{1}\left(f_{0}{ }^{\left(1^{\prime}\right)} f_{0}{ }^{\left(2^{\prime}\right)}-f_{0}{ }^{(p)} f_{0}^{(1)}\right)\right] .
\end{aligned}
$$

Especially the last term contributes to the density dependence of the collisional operator. The others are $n$-dependent via (8). In addition to the usual Chapman-Enskog theory, the integral equation (11) for $f_{1}$ has a density corrected integral kernel. Also the inhomogenity at the l.h.s. contains density modifications. Consequently the solution $f_{1}$ of $(11)$ is the well-known function for $n \rightarrow 0$, modified by density corrections. Thus one gets the correct transport coefficients of dilute gases plus terms $\sim n$. Resigning possible accuracy we now employ the approximation

$$
L\left(f_{1}\right) \approx-f_{1} / \tau \text {. }
$$

$\tau$ must be of order of the time of free flight, averaged over $p$.

$$
\begin{gathered}
\tau^{-1} \overline{\overline{D f}}\left\langle\int \sigma\left(p p_{1} \rightarrow p^{\prime} p_{1}{ }^{\prime} ; f\right) f_{0}\left(p_{1}\right) \mathrm{d} p_{1} \mathrm{~d} p^{\prime} \mathrm{d} p_{1}{ }^{\prime}\right\rangle_{p}, \\
\frac{1}{\tau n}=\left\langle\int \sigma\left(p p_{1} \rightarrow p^{\prime} p_{1}{ }^{\prime} ; f\right) \mathrm{d} p^{\prime} \mathrm{d} p_{1}{ }^{\prime}\right\rangle_{p, p_{1}},
\end{gathered}
$$

$\tau n$ depends on density via the averaging functions $f_{0}$ and because the total cross-section

$$
G(p, q ; f)=\int \sigma\left(p, q \rightarrow p^{\prime} q^{\prime} ; f\right) \mathrm{d} p^{\prime} \mathrm{d} q^{\prime}
$$

does it. In binary collision approximation only the energy conservation law depends on $f$. Triple collisions can be taken care of by the $f$-dependence of the partial cross section.

$$
\frac{1}{\tau n}=\left(\frac{1}{\tau n}\right)_{n=0}\left[1+\frac{\left.(\mathrm{d}\langle G\rangle / \mathrm{d} n)\right|_{n=0}}{\langle G\rangle_{n=0}} n+\ldots\right] .
$$

Thus

$$
\tau n=(\tau n)_{n=0}\left[1+\Delta_{\mathrm{c}} n+\ldots\right]
$$

with

$$
\Delta_{\mathrm{c}}=-\left(\frac{\mathrm{d}}{\mathrm{d} n} \ln \langle G(p, q)\rangle\right)_{n=0} .
$$

We will show in section II that $\Delta_{\mathrm{c}} \sim-B$, thus giving corrections in the same order of magnitude as the corrections in the streaming operator.

With the simplification $\left(6^{\prime}\right)$ the solution of the BoltzmanN-Landau-equation is

$f=f_{0}-\tau\left(\frac{\partial}{\partial t}+\frac{\partial \varepsilon_{p}\left(f_{0}\right)}{\partial \boldsymbol{p}} \cdot \frac{\partial}{\partial \boldsymbol{r}}-\frac{\partial \varepsilon_{p}\left(f_{0}\right)}{\partial \boldsymbol{r}} \cdot \frac{\partial}{\partial \boldsymbol{p}}\right) f_{0}$.

The $\boldsymbol{p}$-dependence of $f(\boldsymbol{r}, \boldsymbol{p}, t)$ is fully determined by (8) and (1), space and time variables enter through $\alpha, \beta, \boldsymbol{u}$. These functions now shall be chosen such, that

$$
\begin{gathered}
n \equiv \int f(\boldsymbol{r}, \boldsymbol{p}, t) \mathrm{d} \boldsymbol{p} \doteq \int f_{0}(\boldsymbol{r}, \boldsymbol{p}, t) \mathrm{d} \boldsymbol{p}, \\
\boldsymbol{u}=\left\langle\frac{\boldsymbol{p}}{m}\right\rangle_{f} \stackrel{!}{=}\left\langle\frac{\boldsymbol{p}}{m}\right\rangle_{f_{0}}=\left\langle\frac{\partial \varepsilon_{p}\left(f_{0}\right)}{\partial \boldsymbol{p}}\right\rangle_{f_{0}} .
\end{gathered}
$$

Consequently it should be

$$
\begin{aligned}
0 & =\int f_{1}(p) \mathrm{d} p \\
& =-\tau \int\left(\frac{\partial}{\partial t}+\frac{\partial \varepsilon_{p}\left(f_{0}\right)}{\partial \boldsymbol{p}} \cdot \frac{\partial}{\partial \boldsymbol{r}}-\frac{\partial \varepsilon_{p}\left(f_{0}\right)}{\partial \boldsymbol{r}} \cdot \frac{\partial}{\partial \boldsymbol{p}}\right) f_{0}(p) \mathrm{d} p \\
& =-\tau\left(\frac{\partial n}{\partial t}+\operatorname{div} n \boldsymbol{u}\right) .
\end{aligned}
$$


This is indeed so, because the continuity equation holds. Similarly one proves

$$
\int \frac{\boldsymbol{p}}{m} f_{1} \mathrm{~d} \boldsymbol{p}=0 .
$$

For: if $\beta$ is chosen as physical temperature, (20) is just the conservation law for the momentum in local equilibrium, see e. g. ${ }^{8}$. [For the last equality sign in (18) see also ${ }^{8}$.]

With the solution (16) of the Boltzmann-Landau equation all interesting quantities can be calculated. At first we consider the stress tensor ${ }^{8}$ for $v \neq \mu$ :

$$
P_{\nu \mu}=\int\left(p_{v}-m u_{v}\right)\left(\frac{\partial \varepsilon_{p}(f)}{\partial p_{\mu}}-u_{\mu}\right) f(p) \mathrm{d} \boldsymbol{p}, \quad v \neq \mu .
$$

If $f=f_{0}$, there would not be any non-diagonal elements; the pressure is isotropic in quasi-equilibrium. Thus

$$
\begin{aligned}
P_{v \mu} & =\int\left(p_{v}-m u_{v}\right)\left(\frac{\partial \varepsilon_{p}\left(f_{0}\right)}{\partial p_{\mu}}-u_{\mu}\right) f_{1} \mathrm{~d} \boldsymbol{p} \\
& +\int\left(p_{v}-m u_{v}\right) \frac{\partial}{\partial p_{\mu}} F(\boldsymbol{p}, \boldsymbol{q}) f_{1}(\boldsymbol{q}) f_{0}(\boldsymbol{p}) \mathrm{d} \boldsymbol{p} \mathrm{d} \boldsymbol{q} \\
& +\sim f_{1}{ }^{2} .
\end{aligned}
$$

One might ask, whether $P_{\nu \mu}$ is a symmetric tensor. This can be proved to be so. But the explicit calculation of (21) in section III will give

$$
P_{\nu \mu} \sim\left(\frac{\partial u_{v}}{\partial x_{\mu}}+\frac{\partial u_{\mu}}{\partial x_{v}}\right) \quad(v \neq \mu),
$$

thus showing directly the symmetry of the stress tensor. If one furthermore defines the constant of proportionality as shear viscosity $\eta$, the density corrections of $\eta$ are included in the result.
At next we are interested in the energy flow

$$
\boldsymbol{Q}=\int \varepsilon_{p}(f) \frac{\partial \varepsilon_{p}(f)}{\partial \boldsymbol{p}} f(\boldsymbol{p}) \mathrm{d} \boldsymbol{p}=\boldsymbol{Q}_{0}+\boldsymbol{Q}_{1}+\sim f_{1}{ }^{2}
$$

As has been derived in ${ }^{8}$, in local equilibrium energy transport only occurs by convection

$$
\boldsymbol{Q}_{0}=\boldsymbol{u}\left(P+\varepsilon+\frac{1}{2} n m u^{2}\right) .
$$

In section IV we will show $\boldsymbol{Q}_{1} \sim(\partial / \partial \boldsymbol{r}) T$, the constant of proportionality being defined as (density dependent) coefficient of thermal conductivity, $\lambda$.

\section{Density Dependence of the Time of Mean Free Flight}

In the approximation $\left(6^{\prime}\right)$ of the usual BoltzMANN equation the transport coefficients may be shown to be

$$
\begin{gathered}
\eta=\tau n \varkappa \mathrm{T}, \\
\lambda=\frac{5}{2} \tau n\left(\varkappa^{2} T / m\right)=\frac{5}{3}\left(c_{v} / m\right) \eta .
\end{gathered}
$$

This follows from (21) and (22) with $F=0$ in the streaming operator. For the proof one may either look in the textbook of HUANG ${ }^{10}$ or wait until later, formulae (38) and (50). Without quantum corrections of the streaming term in (5) all we had to do would be to take care of the density dependence of $\tau$ according to (14) :

$$
\begin{gathered}
\eta=\eta_{0}\left(1+\Delta_{\mathrm{c}} n\right), \\
\lambda / \eta=\lambda_{0} / \eta_{0}=\frac{5}{3} c_{v} / m .
\end{gathered}
$$

More exact calculations give for $\lambda / \eta$ a factor very near $\frac{5}{2}$ instead of $\frac{5}{3}^{4}$, showing that our simplification $(6)$ may be erroneous at least $\approx 40 \%$. Let us now determine $\Delta_{\mathrm{c}}$ from (15).

$$
\begin{aligned}
\langle G\rangle_{n=0} & =\left\langle\int \hat{\sigma}\left(p p_{1} ; p^{\prime} p_{1}^{\prime}\right) \delta\left(p+p_{1}-p^{\prime}-p_{1}{ }^{\prime}\right) \delta\left(\frac{p^{2}}{2 m}+\ldots\right) \mathrm{d} p^{\prime} \mathrm{d} p_{1}^{\prime}\right\rangle \text { free Maxw. distrib. } \\
& =\left\langle\int \frac{1}{2} m|k| \hat{\sigma}\left(|k|, \omega^{\prime} ; K\right) \mathrm{d} \omega^{\prime}\right\rangle_{k, K} .
\end{aligned}
$$

The connection between the usual total cross section and $\hat{\sigma}$ is

$$
\left(\frac{m}{2}\right)^{2} \int \hat{\sigma}\left(|k|, \omega^{\prime} ; K\right) \mathrm{d} \omega^{\prime} \overline{\overline{\mathrm{Df}}} \sigma_{\mathrm{tot}}(|k|), \text { independent from total momentum. }
$$

Thus

$$
(\tau n)_{n=0}^{-1}=\langle G(p, q ; f=0)\rangle_{n=0}=\left\langle\frac{|k|}{\frac{1}{2} m} \sigma_{\text {tot }}(|k|)\right\rangle_{k}=\left\langle v_{\text {rel }} \sigma_{\text {tot }}\left(v_{\text {rel }}\right)\right\rangle .
$$

As $G(p, q ; f=0) \sim \sigma_{\text {tot }}(p-q)$ only depends on the relative momentum $k,(\tau n)_{n=0}$ is independent from $\boldsymbol{u}$. The result is the wellknown formula $l_{0} \approx 1 / n \sigma_{\text {tot }}$. The $K$-independence of $G$, the imaginary part of the forward scattering amplitude corresponds to that of $F$, the real part. Now the next order of density:

$\frac{\mathrm{d}}{\mathrm{d} n}\langle G(p, q)\rangle_{n=0}=\int \mathrm{d} p \mathrm{~d} q G(p, q ; f=0)\left(\frac{\mathrm{d}}{\mathrm{d} n} w(p) w(q)\right)_{n=0}+\int \mathrm{d} p \mathrm{~d} q w_{0}(p) w_{0}(q)\left(\frac{\mathrm{d}}{\mathrm{d} n} G(p, q ; f)\right)_{n=0}$

with the normalized momentum distribution:

$$
w_{0}(p)=\left(\frac{\exp \left\{\beta\left(\alpha+\boldsymbol{p} \cdot \boldsymbol{u}-\varepsilon_{p}\left(f_{0}\right)\right\}\right.}{\int \exp \left\{\beta\left(\alpha+\boldsymbol{p} \cdot \boldsymbol{u}-\varepsilon_{p}\left(f_{0}\right)\right\} \mathrm{d} \boldsymbol{p}\right.}\right)_{n=0}=\frac{\exp \left\{-(\beta / 2 m)(\boldsymbol{p}-m \boldsymbol{u})^{2}\right\}}{\int \exp \left\{-(\beta / 2 m)(\boldsymbol{p}-m \boldsymbol{u})^{2}\right\} \mathrm{d} \boldsymbol{p}} .
$$


The first term in (28) is easily to be calculated with the methods, shown in the appendix of ${ }^{8}$. The result is

$$
-2 \beta\{G(\bar{p}, \bar{q} ; f=0) F(\bar{q}, \bar{s})-G(\bar{p}, \bar{q} ; f=0) F(\bar{s}, t)\} .
$$

The dash indicates the mean value with the free Maxwellian distribution (29). As $G(f=0)$ and $F$ only depend on the relative momenta, this result does not depend on $\boldsymbol{u}$. If we assume $G$ and/or $\boldsymbol{F}$ approximately to be independent from $p-q$, the correlated mean value factorizes and this contribution to $\Delta_{\mathrm{c}}$ vanishes. It is of order of $C$ (third virial coefficient), see ${ }^{8}$.

The second term in (28) is the average of $\quad\left(\frac{\mathrm{d}}{\mathrm{d} n} G\left(\frac{K}{2}+k, \frac{K}{2}-k ; f\right)\right)_{n=0}$, weighed with the free distributions $\frac{\exp \left\{-\beta k^{2} / m\right\}}{\int \exp \left\{-\beta k^{2} / m\right\} \mathrm{d} \boldsymbol{k}} \cdot \frac{\exp \left\{-(\beta / 4 m)(\boldsymbol{K}-2 m \boldsymbol{u})^{2}\right\}}{\int \exp \left\{-(\beta / 4 m)(\boldsymbol{K}-2 m \boldsymbol{u})^{2}\right\} \mathrm{d} \boldsymbol{K}}$.

Transforming the $\boldsymbol{K}$-average around $\boldsymbol{u}=0$ and indicating again such averaging procedure by a dash, we have

$$
\left\langle\int \hat{\sigma}\left(\boldsymbol{k}, \boldsymbol{k}^{\prime}\right)\left(\frac{\mathrm{d}}{\mathrm{d} n} \delta\left(E_{k}-E_{k^{\prime}}\right)\right)_{n=0} \mathrm{~d} \boldsymbol{k}^{\prime}\right\rangle_{k}
$$

where

$$
\begin{aligned}
E_{k} & =\frac{k^{2}}{m}+n \int\left[F\left(k, q-\frac{\bar{K}}{2}-m u\right)+F\left(-k, q-\frac{\bar{K}}{2}-m u\right)\right] w_{0}(q) \mathrm{d} q \\
\quad & =\frac{k^{2}}{m}+n\left(F\left(k, \bar{q}-\frac{\bar{K}}{2}\right)+F\left(-k, \bar{q}-\frac{\bar{K}}{2}\right)\right) .
\end{aligned}
$$

Originally the averaging over $K$ should be performed outside the energy conservation law. But in lowest order in the density only terms linear in $n F$ are taken care of in the conservation law. This allows the averaging procedure under the $\delta$-function. As consequence we see that $E_{k}$ only depends from $|k|$.

$$
\begin{aligned}
\delta\left(E_{k}\right. & \left.-E_{k^{\prime}}\right)=\delta\left(\left(k-k^{\prime}\right) \frac{\mathrm{d} E_{k}}{\mathrm{~d} k}\right) \\
& =\delta\left(k-k^{\prime}\right) \frac{m}{2 k}\left(1-\frac{m}{k} n \frac{\mathrm{d}}{\mathrm{d} k} F\left(k, \bar{q}-\frac{\bar{K}}{2}\right)\right) .
\end{aligned}
$$

Now the derivation with respect to $n$ can be performed.

$$
\begin{aligned}
& -\left\langle\int \hat{\sigma}\left(k, \omega^{\prime}\right) \mathrm{d} \omega^{\prime} \frac{m^{2}}{2} \frac{\mathrm{d}}{\mathrm{d} k} \boldsymbol{F}\left(k, \bar{q}-\frac{\bar{K}}{2}\right)\right\rangle \\
& =-2\left\langle\sigma_{\text {tot }}(k) \frac{\mathrm{d}}{\mathrm{d} k} \boldsymbol{F}\left(k, \bar{q}-\frac{\bar{K}}{2}\right)\right\rangle \\
& =2\left\langle\boldsymbol { F } ( k , \overline { q } - \frac { \overline { K } } { 2 } ) \left[\frac{\partial}{\partial \boldsymbol{k}}\left(\frac{\boldsymbol{k}}{k} \sigma_{\mathrm{tot}}(k)\right)\right.\right. \\
& \left.\quad+\sigma_{\text {tot }} \frac{\boldsymbol{k}}{k}\left(-\frac{\beta 2 \boldsymbol{k}}{m}\right)\right\rangle_{k} \\
& =2\left\langle\boldsymbol{F}\left(k, \bar{q}-\frac{\bar{K}}{2}\right)\left[\frac{m}{k^{2}}-\beta\right] G(k ; f=0)\right\rangle_{k} \\
& +2\left\langle\boldsymbol{F}\left(k, \bar{q}-\frac{K}{2}\right) \frac{\mathrm{d} \sigma_{\mathrm{tot}}}{\mathrm{d} k}\right\rangle_{k}
\end{aligned}
$$

Term (30) and term (31) together yield $\Delta_{\mathrm{c}}$ after division by (27). If the cross section only slowly depends from $k$ and the correlated averages may be factorized, the approximate result is

$$
\Delta_{\mathrm{c}}=-2\left\langle\left(\frac{m}{k^{2}}-\beta\right) F\left(k, \bar{q}-\frac{\bar{K}}{2}\right)\right\rangle_{k} .
$$

Again some knowledge of $F$ would be needed. With-

\begin{tabular}{|c|c|c|c|c|c|}
\hline Gas & $\begin{array}{c}\delta \\
\AA^{3}\end{array}$ & $\frac{\delta}{B}$ & $\begin{array}{l}\varepsilon / \varkappa \\
{ }^{\circ} \mathrm{K}\end{array}$ & $T^{*}=\varkappa T / \varepsilon$ & $\begin{array}{l}b_{0} \\
\AA^{3}\end{array}$ \\
\hline $\begin{array}{l}\mathrm{A} \\
\mathrm{Ne} \\
\mathrm{N}_{2} \\
\mathrm{O}_{2}\end{array}$ & $\begin{array}{c}31 \\
4.5 \\
28 \\
24\end{array}$ & $\begin{array}{r}-1.09 \\
0.24 \\
-3.14 \\
-0.89\end{array}$ & $\begin{array}{r}120 \\
35 \\
95 \\
118\end{array}$ & $\begin{array}{l}2.44 \\
8.36 \\
3.08 \\
2.48\end{array}$ & $\begin{array}{r}83 \\
45 \\
106 \\
87\end{array}$ \\
\hline
\end{tabular}
out details we only can conclude [with (7)]

$$
\Delta_{\mathrm{c}} \sim-B \text {. }
$$

This fairly agrees with some experimental results at $20^{\circ} \mathrm{C}$.

Take the experimental law $\eta=\eta_{0}(1+n \delta)$. Then $\delta$ is determined from ${ }^{7}$, p. $2.227 / 8$. The virial coefficient is taken from ${ }^{11}, \S 3.6$ according to the LenNard-Jones $(6-12)$ potential, which agrees very good with experimental values of $B(T)$. It is $B=b_{0} B^{*}\left(T^{*}\right)$; the parameters are given, $B^{*}\left(T^{*}\right)$ is tabulated in ${ }^{11}$, p. 1114.

11 J. O. Hirschfelder, C. F. Curtiss, and R. B. Bird, Molecular Theory of Gases and Liquids, Interscience Publ. Inc., London-New York 1954. 


\section{Coefficient of Viscosity}

Now we'll determine the density dependence of $\eta$ on account of streaming effects, which add to (33). To do this we use the expression (21) for the non-diagonal elements of the pressure tensor together with the solution (16) of the Boltzmann-Landau equation, accomplished by (17), (18). It is

$$
f_{1}=-\tau f_{0}\left(\frac{\partial}{\partial t}+\frac{\partial \varepsilon_{p}\left(f_{0}\right)}{\partial \boldsymbol{p}} \cdot \frac{\partial}{\partial \boldsymbol{r}}-\frac{\partial \varepsilon_{p}\left(f_{0}\right)}{\partial \boldsymbol{r}} \cdot \frac{\partial}{\partial \boldsymbol{p}}\right)\left(\beta \alpha+\beta \boldsymbol{p} \cdot \boldsymbol{u}-\beta \varepsilon_{p}\left(f_{0}\right)\right) .
$$

Using

$$
\left(\frac{\partial \varepsilon_{p}}{\partial p_{\mu}}-u_{\mu}\right) f_{0}=-\varkappa T \frac{\partial}{\partial p_{\mu}} f_{0}
$$

in the first part of (21), integration by part and watching $\nu \neq \mu$ we have

$$
\frac{1}{-\tau n \varkappa T} P_{\nu \mu}=\left\langle\left(p_{v}-m u_{\nu}\right) \frac{\partial}{\partial p_{\mu}}\left(\frac{\partial}{\partial t}+\frac{\partial \varepsilon_{p}\left(f_{0}\right)}{\partial \boldsymbol{p}} \cdot \frac{\partial}{\partial \boldsymbol{r}}-\frac{\partial \varepsilon_{p}\left(f_{0}\right)}{\partial \boldsymbol{r}} \cdot \frac{\partial}{\partial \boldsymbol{p}}\right)\left(\beta \alpha+\beta \boldsymbol{p} \cdot \boldsymbol{u}-\beta \varepsilon_{p}\left(f_{0}\right)\right)\right\rangle_{f_{0}}+\Pi_{\nu \mu} .
$$

$\Pi_{v \mu}$ is the second part in (21) and will now be shown to vanish. Integration by part, using (35), and omitting all terms $\sim F$ because $\Pi_{\nu \mu}$ explicitly is proportional to $F$ :

$$
\Pi_{v \mu} \sim\left\langle\left(p_{v}-m u_{v}\right)\left(p_{\mu}-m u_{\mu}\right) F(p, q)\left(\frac{\partial}{\partial t}+\frac{\boldsymbol{q}}{m} \cdot \frac{\partial}{\partial \boldsymbol{r}}\right) \beta\left(\alpha+\boldsymbol{q} \cdot \boldsymbol{u}-\frac{1}{2 m} q^{2}\right)\right\rangle_{w_{0}(p) w_{0}(q)} .
$$

For the same reason $w_{0}$ from (29) has to be used for the averaging procedure. We now transform in momentum space to $\boldsymbol{u}=0$; that does not influence $F$ because (2).

$$
\Pi_{v \mu} \sim\left\langle p_{\nu} p_{\mu} F(p, q)\left[\left(\frac{\partial}{\partial t}+\boldsymbol{u} \cdot \frac{\partial}{\partial \boldsymbol{r}}\right)+\frac{\boldsymbol{q}}{m} \cdot \frac{\partial}{\partial \boldsymbol{r}}\right]\left[\beta\left(\alpha+\frac{m}{2} u^{2}\right)-\frac{\beta}{2 m} q^{2}\right]\right\rangle_{0} .
$$

Evidently this average in the isotropic free MAxweLL distribution vanishes because odd moments are taken as $v \neq \mu$. In the remaining expression for $P_{\nu \mu}$ most of the terms vanish by similar arguments.

$$
\begin{gathered}
\left\langle\left(p_{v}-m u_{v}\right) \frac{\partial}{\partial p_{u}} \frac{\partial \beta a}{\partial t}\right\rangle=0, \quad \text { evidently, }\left\langle\left(p_{v}-m u_{v}\right) \frac{\partial}{\partial t} \beta u_{\mu}\right\rangle=0, \text { because (18), } \\
\left\langle\left(p_{v}-m u_{v}\right) \frac{\partial}{\partial t}\left(\beta \frac{\partial \varepsilon_{p}}{\partial p_{\mu}}\right)\right\rangle=0, \quad \text { namely, }\left\langle\left(p_{v}-m u_{v}\right)\left(\frac{\partial \varepsilon_{p}}{\partial p_{u}}-u_{\mu}+u_{\mu}\right)\right\rangle=0, \quad \text { evidently, }
\end{gathered}
$$

and

$$
\left\langle\left(p_{v}-m u_{v}\right) \frac{\partial}{\partial p_{u}} F(p, q) \frac{\partial}{\partial t}\left(\beta \alpha+\beta \boldsymbol{q} \cdot \boldsymbol{u}-\beta \frac{1}{2 m} q^{2}\right)\right\rangle=0, \text { similar to } \Pi_{v \mu} .
$$

Furthermore $\left\langle\left(p_{v}-m u_{v}\right) \frac{\partial}{\partial p_{u}} \frac{\partial \varepsilon_{p}}{\partial \boldsymbol{p}}\right\rangle \cdot \frac{\partial \beta \alpha}{\partial \boldsymbol{r}}=0 \quad$ because $\quad \frac{\partial^{2} \varepsilon_{p}}{\partial p_{u} \partial p_{\lambda}}=\frac{1}{m} \delta_{\mu \lambda}+n \frac{\partial^{2}}{\partial p_{u} \partial p_{\lambda}} F(p, \bar{q})$.

The first term does not contribute because (18); in the second one transformation to $\boldsymbol{u}=0$ is possible, $F(p, \bar{q})$ then only depends from $|p|$, showing the average to be an odd moment.

$$
\left\langle\left(p_{v}-m u_{v}\right) \frac{\partial}{\partial p_{\mu}}\left(-\frac{\partial \varepsilon_{p}}{\partial \boldsymbol{r}} \cdot \frac{\partial}{\partial \boldsymbol{p}}\right) \beta \alpha\right\rangle=0, \text { evidently. }
$$

At last

$$
\left\langle\left(p_{v}-m u_{v}\right) \frac{\partial}{\partial p_{\mu}}\left(-\frac{\partial \varepsilon_{p}}{\partial \boldsymbol{r}}\right) \cdot \beta \boldsymbol{u}\right\rangle=-n \beta \boldsymbol{u} \cdot\left\langle\left(p_{v}-m u_{v}\right) \frac{\partial}{\partial p_{\mu}} F(p, q) \frac{\partial}{\partial \boldsymbol{r}}\left(\beta \alpha+\beta \boldsymbol{q} \cdot \boldsymbol{u}-\frac{\beta}{2 m} q^{2}\right)\right\rangle=0 .
$$

The remaining contributions are

$$
\begin{aligned}
& \left\langle\left(p_{v}-m u_{v}\right) \frac{\partial}{\partial p_{u}}\left\{\frac{\partial \varepsilon_{p}}{\partial \boldsymbol{p}} \cdot \frac{\partial}{\partial \boldsymbol{r}}(\beta \boldsymbol{p} \cdot \boldsymbol{u})-\frac{\partial \varepsilon_{p}}{\partial \boldsymbol{p}} \cdot \frac{\partial}{\partial \boldsymbol{r}}\left(\beta \varepsilon_{p}\right)+\frac{\partial \varepsilon_{p}}{\partial \boldsymbol{r}} \cdot \frac{\partial}{\partial \boldsymbol{p}}\left(\beta \varepsilon_{p}\right)\right\}\right\rangle \\
= & \left\langle\left(p_{v}-m u_{v}\right) \frac{\partial}{\partial p_{\mu}} \frac{\partial \varepsilon_{p}}{\partial \boldsymbol{p}}\left(p \cdot \boldsymbol{u}-\varepsilon_{p}\right)\right\rangle \cdot \frac{\partial \beta}{\partial \boldsymbol{r}}+\beta\left\langle\left(p_{v}-m u_{v}\right) \frac{\partial}{\partial p_{\mu}} \frac{\partial \varepsilon_{p}}{\partial \boldsymbol{p}} \cdot \frac{\partial}{\partial \boldsymbol{r}}(\boldsymbol{p} \cdot \boldsymbol{u})\right\rangle .
\end{aligned}
$$

It is shown by similar arguments as above, that the factor before $\partial \beta / \partial \boldsymbol{r}$ vanishes too. Thus

$$
\begin{aligned}
\frac{1}{-\tau n \varkappa T} P_{\nu \mu} & =\beta \sum_{\lambda, \varrho} \frac{\partial u_{\varrho}}{\partial x_{\lambda}}\left\langle\left(p_{v}-m u_{v}\right)\left[\frac{\partial^{2} \varepsilon_{p}}{\partial p_{\mu} \partial p_{\lambda}} p_{\varrho}+\frac{\partial \varepsilon_{p}}{\partial p_{\lambda}} \delta_{\mu \varrho}\right]\right\rangle \\
& =\beta \sum_{\lambda, \varrho} \frac{\partial u_{\varrho}}{\partial x_{\lambda}}\left(\delta_{\mu \lambda} \delta_{\nu \varrho}+\delta_{\mu \varrho} \delta_{\nu \lambda}\right)\left[\varkappa T+n\left\langle p_{\nu}{ }^{2} p_{\mu}{ }^{2}\left(\frac{F^{\prime \prime}}{p^{2}}-\frac{F^{\prime}}{p^{3}}\right)\right\rangle\right] \\
& =\left(\frac{\partial u_{\mu}}{\partial x_{\nu}}+\frac{\partial u_{v}}{\partial x_{\mu}}\right)\left[1+n \beta\left\langle p_{\nu} p_{\mu} \frac{\partial^{2}}{\partial p_{\nu} \partial p_{\mu}} F(p, q)\right\rangle_{0}\right] .
\end{aligned}
$$


Here has been used that $n \int F(p, q) w_{0}(q) \mathrm{d} q$ depends on $|p|$ only (after transformation to $\boldsymbol{u}=0$ ). We thus found

$$
P_{\nu \mu} \equiv-\eta\left(\frac{\partial u_{v}}{\partial x_{\mu}}+\frac{\partial u_{u}}{\partial x_{\nu}}\right)=P_{\mu \nu} \quad(\nu \neq \mu)
$$

with the constant of proportionality

$$
\begin{gathered}
\eta=\tau n \varkappa T\left(1+n \Delta_{\mathrm{s}}{ }^{\eta}\right), \\
\Delta_{\mathrm{s}}{ }^{\eta \overline{\mathrm{Df}}} \beta\left\langle p_{\nu} p_{\mu} \frac{\partial^{2}}{\partial p_{\nu} \partial p_{\mu}} F\right\rangle_{0} .
\end{gathered}
$$

The pressure tensor is symmetric as stated before. The viscosity $\eta$ will be shown as independent of $v, \mu$, therefore being a pure number. In the low density limit $n \Delta_{\mathrm{s}}{ }^{\eta} \rightarrow 0$ the statement (23) is proved. We only need to calculate now the first virial correction $\Delta_{\mathrm{s}}{ }^{\eta}$ of the viscosity, which arises from the modification of the streaming operator in the Boltzmann-Landau-equation. The free Maxwellian average (39) can be reduced to the virial coefficient and its derivatives. Introducing relative and total momentum and taking care of (2) gives

$$
\begin{aligned}
\Delta_{\mathrm{s}}{ }^{\eta} & =\beta \frac{1}{4}\left\langle\left(\frac{K_{\nu}}{2}+k_{\nu}\right)\left(\frac{K_{\mu}}{2}+k_{\mu}\right) \frac{\partial^{2}}{\partial k_{\nu} \partial k_{\mu}} F(|k|)\right\rangle_{0} \\
& =\frac{\beta}{4}\left\langle k_{\nu} k_{\mu} \frac{\partial^{2}}{\partial k_{\nu} \partial k_{\mu}} F(|k|)\right\rangle_{0} .
\end{aligned}
$$

Now integrate by parts:

$$
\Delta_{\mathrm{s}}^{\eta}=\frac{\beta}{4}\left\langle F-\frac{2 \beta}{m} \cdot \frac{1}{3} k^{2} F \cdot 2+\left(\frac{2 \beta}{m}\right)^{2} k_{\nu}{ }^{2} k_{\mu}{ }^{2} F\right\rangle_{0} .
$$

One easily proves the formulae

$$
\begin{aligned}
\left\langle\left(\frac{k_{v}}{k}\right)^{2}\left(\frac{k_{\mu}}{k}\right)^{2}\right\rangle_{\omega} & =\frac{1}{15} \quad(\nu \neq \mu), \\
\left\langle\frac{\beta}{2} F \beta \frac{k^{2}}{m}\right\rangle_{0} & =\frac{5}{2}\left(B+\frac{2}{5} T B^{\prime}\right), \\
\left\langle\frac{\beta}{2} F\left(\beta \frac{k^{2}}{m}\right)^{2}\right\rangle_{0}= & \frac{35}{4} B+7 T B^{\prime}+T^{2} B^{\prime \prime},
\end{aligned}
$$

following from (7). This leads to the result

$$
\Delta_{\mathrm{s}}^{\eta}=\frac{2}{15}\left(2 T B^{\prime}+T^{2} B^{\prime \prime}\right)=\frac{2}{15} \frac{\mathrm{d}}{\mathrm{d} T}\left(T^{2} B^{\prime}\right) \text {. }
$$

As $\Delta_{\mathrm{s}}{ }^{\eta}$ contains derivatives of $B$, it tests higher moments of $F(k)$. It therefore is a sensitive test of the intermolecular forces. For the (too simple) VAN DER WAALS model for $B$ we get

$$
\Delta_{\mathrm{s}}{ }^{\eta}\left(\text { van DER } \mathrm{W}_{\text {AaLS }}\right)=0 .
$$

E. g. neither a weak interacting $(B=a / \varkappa T)$ nor a classical hard core gas $(B=$ const $)$ give a streaming correction to the virial coefficient of the viscosity. But with the empirical $B(T)$, being represented excellently by the LenNARD-Jones-model (ref. ${ }^{11}$, $\S 3.6$ ) we get the following results.

\begin{tabular}{|l|c|c|}
\hline Gas & $\frac{\Delta_{\mathrm{s}} \eta}{B}=\frac{2}{15} \frac{2 B_{1}{ }^{*}\left(T^{*}\right)+B_{2}{ }^{*}\left(T^{*}\right)}{B_{0}{ }^{*}\left(T^{*}\right)}$ & $\frac{\Delta_{\mathrm{s}} \eta}{\delta}$ \\
\hline $\mathrm{A}$ & 0.149 & $-13.7 \%$ \\
$\mathrm{Ne}$ & -0.042 & $-17.5 \%$ \\
$\mathrm{~N}_{2}$ & 0.40 & $-12.7 \%$ \\
$\mathrm{O}_{2}$ & 0.152 & $-17.1 \%$ \\
\hline
\end{tabular}

The comparison with the experimental values shows that $\Delta_{\mathrm{s}}{ }^{\eta}$ tends to diminish the effect of $\Delta_{\mathrm{c}} \sim \delta$ about approximately $15 \%$. Thus in first approximation ${ }^{12}$ the density dependence of the collisional operator, giving (33), explains the virial coefficient of $\eta$; but more accurate evaluations of (32), (31) or of the exact integral kernel $\left(6^{\prime}\right)$ will probably make it necessary to take care of the streaming corrections in the Boltzmann-Landau-equation.

Perhaps it is interesting to compare our results

\begin{tabular}{|c|c|c|c|c|}
\hline Gas & A & $\mathrm{Ne}$ & $\mathrm{N}_{2}$ & $\mathrm{O}_{2}$ \\
\hline$\eta($ ENSKOG $) \AA^{3}$ & 14.5 & 7.88 & 18.6 & 15.2 \\
\hline
\end{tabular}
with those of ENskog 4, 11. Enskog's theory gives the virial expansion

$$
\begin{array}{ll} 
& \eta=\eta_{0}\left(1+\frac{7}{40} B n+\ldots\right), \\
\text { i. e. } \quad \Delta^{\eta}(\text { ENSKOG }) / B=0.175 .
\end{array}
$$

That would be of the same magnitude as our $\Delta_{s} \eta$, if the empirical values of $B$ were taken. But if - what would be more consequent in ENskog's theory of hard spheres - the hard sphere values of

$$
B=\frac{2}{3} \pi \sigma^{3}=b_{0}
$$

would be taken, we get the values:

These are too small (except for $\mathrm{Ne}$ ), but - what is worse-are independent of temperature. As the consequent theory of hard spheres not all agreed with experiment, ENskog used some ad hoc modification, which for moderately dense gases gives

$$
\tilde{\Delta}^{\eta}(\text { ENskog })=0.175\left(B+T B^{\prime}\right)
$$

where now empirical values of $B$ should be taken.

\begin{tabular}{c|cccc} 
Gas & $\mathrm{A}$ & $\mathrm{Ne}$ & $\mathrm{N}_{2}$ & $\mathrm{O}_{2}$ \\
\cline { 2 - 6 }$\Delta^{\eta}($ ENSKOG $) / B$ & -0.46 & 0.28 & -1.63 & -0.49
\end{tabular}

This is also too small (except Ne). Indeed Enskog got fairly good agreement with some experimental

12 The experimental values of $\delta$ for $\mathrm{N}_{2}$ disagree about 20\% as given in ${ }^{4}$ or ${ }^{7}$. This should only partially be due to the different temperatures. 
results not until he fitted the hard sphere parameter $\sigma$ at the empirical plot; but if one fits in (44), (45), the absolut value of the first virial correction is right per constructionem. All that could be checked is the dependence from temperature. But there are no experimental results available.

To sum up our results: The transport theory of Chapman and Enskog applied to the BoltzmannLANDAU-equation in binary collision approximation seems to deliver a method for calculating the density dependence of the transport coefficients, here especially of the shear viscosity. The magnitude of the virial correction of $\eta$ can be explained. The method seems to be a natural extension of the ENskog theory for dense gases at moderate densities, but without its severe disadvantages: (5) holds for rather arbitrary intermolecular forces and not only for hard spheres; no ad hoc modification is needed to correct the results; no fitting is needed. But in contrast, the disadvantage of the theory presented here is - besides the large numerical efforts of a more exact solution, but which is met in other methods ${ }^{2,3}$ too - that one needs some more knowledge about the real part of the forward scattering amplitude, $F$, and $\sigma$, which possibly goes beyond $B$, $B^{\prime}, \ldots$ Thus a predominate task is to get that knowledge first!

\section{Coefficient of Thermal Conductivity}

Now the heat flow vector $\boldsymbol{Q}$ shall be determined from (22). If we consider a physical situation, in which

$$
\boldsymbol{u}(\boldsymbol{r}, t) \equiv 0,
$$

no energy transport by convection occurs: $\boldsymbol{Q}_{\mathbf{0}}=0$. The energy transport by conduction is $\sim f_{1} \sim \tau$ :

$$
\begin{aligned}
& \boldsymbol{Q}=\int \varepsilon_{p}\left(f_{0}\right) \frac{\partial \varepsilon_{p}\left(f_{0}\right)}{\partial \boldsymbol{p}} f_{1}(p) \mathrm{d} \boldsymbol{p}+\int \varepsilon_{p}\left(f_{0}\right) f_{0}(p) \frac{\partial}{\partial \boldsymbol{p}} F(p, q) f_{1}(q) \mathrm{d} \boldsymbol{p} \mathrm{d} \boldsymbol{q}+\int F(p, q) f_{1}(q) \frac{\partial \varepsilon_{p}\left(f_{0}\right)}{\partial \boldsymbol{p}} f_{0}(p) \mathrm{d} \boldsymbol{p} \mathrm{d} \boldsymbol{q} \\
&=\int \varepsilon_{p}\left(f_{0}\right) \frac{\partial \varepsilon_{p}\left(f_{0}\right)}{\partial \boldsymbol{p}} f_{1}(p) \mathrm{d} \boldsymbol{p}-\int F(p, q) \varepsilon_{p}\left(f_{0}\right) \frac{\partial f_{0}(p)}{\partial \boldsymbol{p}} f_{1}(q) \mathrm{d} \boldsymbol{p} \mathrm{d} \boldsymbol{q} . \\
& \frac{1}{-\tau n} \boldsymbol{Q}=\left\langle\left(\varepsilon_{p} \frac{\partial \varepsilon_{p}}{\partial \boldsymbol{p}}+\beta n F(p, q) \varepsilon_{q} \frac{\partial \varepsilon_{q}}{\partial \boldsymbol{q}}\right)\left(\frac{\partial}{\partial t}+\frac{\partial \varepsilon_{p}}{\partial \boldsymbol{p}} \cdot \frac{\partial}{\partial \boldsymbol{r}}-\frac{\partial \varepsilon_{p}}{\partial \boldsymbol{r}} \cdot \frac{\partial}{\partial \boldsymbol{p}}\right) \cdot\left(\beta \alpha-\beta \varepsilon_{p}\right)\right\rangle .
\end{aligned}
$$

The weight function for averaging is (8) with $\boldsymbol{u}=0$. Thus $\varepsilon_{p}$ as well as the $p$ - (or $q$-) average of $F(p, q$ ) only depend on $|\boldsymbol{p}|$ ( or $|\boldsymbol{q}|$ ). Consequently some terms vanish by symmetry arguments, what can be checked easily:

$$
\left\langle\left(\varepsilon_{p} \frac{\partial \varepsilon_{p}}{\partial \boldsymbol{p}}+\beta n F(p, q) \varepsilon_{q} \frac{\partial \varepsilon_{q}}{\partial \boldsymbol{q}}\right) \frac{\partial}{\partial t}\left(\beta \alpha-\beta \varepsilon_{p}\right)\right\rangle=0 .
$$

Because $\quad\left(\frac{\partial \varepsilon_{p}}{\partial \boldsymbol{p}} \cdot \frac{\partial}{\partial \boldsymbol{r}}-\frac{\partial \varepsilon_{p}}{\partial \boldsymbol{r}} \cdot \frac{\partial}{\partial \boldsymbol{p}}\right)\left(\beta \alpha-\beta \varepsilon_{p}\right)=\frac{\partial \varepsilon_{p}}{\partial \boldsymbol{p}} \cdot \frac{\partial(\beta \alpha)}{\partial \boldsymbol{r}}-\varepsilon_{p} \frac{\partial \varepsilon_{p}}{\partial \boldsymbol{p}} \cdot \frac{\partial \beta}{\partial \boldsymbol{r}}$,

we have (again using symmetry arguments)

$$
\frac{1}{-\tau n} \boldsymbol{Q}=\left\langle\left(\varepsilon_{p} \frac{\partial \varepsilon_{p}}{\partial \boldsymbol{p}}+\beta n F(p, q) \varepsilon_{q} \frac{\partial \varepsilon_{q}}{\partial \boldsymbol{q}}\right) \cdot \frac{\partial \varepsilon_{p}}{3 \partial \boldsymbol{p}}\left(\frac{\partial(\beta \alpha)}{\partial \boldsymbol{r}}-\varepsilon_{p} \frac{\partial \beta}{\partial \boldsymbol{r}}\right)\right\rangle .
$$

Both terms are $\sim \frac{\partial \beta}{\partial \boldsymbol{r}}=-\beta \frac{1}{T} \frac{\partial T}{\partial \boldsymbol{r}}$. This is a consequence of $\beta \alpha=\ln n+2 n B-\frac{3}{2} \ln T+$ const,

which can be derivated from (9), see ${ }^{8}$ eq. (28). The spatial gradient of $(\beta \alpha)$ also depends on $\partial n / \partial \boldsymbol{r}$, which in turn is connected to the temperature gradient by the momentum conservation law. As $\boldsymbol{u}=0$, the stress tensor remains diagonal and its spatial gradient vanishes:

$$
\frac{\partial}{\partial \boldsymbol{r}}[n \varkappa T(1+n B)]=0, \quad \text { which gives } \frac{\partial(\beta a)}{\partial \boldsymbol{r}}=-\frac{1}{T} \frac{\partial T}{\partial \boldsymbol{r}}\left(\frac{5}{2}+n B-n T B^{\prime}\right) .
$$

We thus get $\boldsymbol{Q} \sim \operatorname{grad} T$, the constant of proportionality being the negative coefficient of thermal conductivity

$$
-\lambda=\frac{1}{3} \tau n \varkappa \beta\left\langle\left(\varepsilon_{p} \frac{\partial \varepsilon_{p}}{\partial \boldsymbol{p}}+\beta n F(p, q) \varepsilon_{q} \frac{\partial \varepsilon_{q}}{\partial \boldsymbol{q}}\right) \cdot \frac{\partial \varepsilon_{p}}{\partial \boldsymbol{p}}\left(\frac{5}{2}+n B-n T B^{\prime}-\beta \varepsilon_{p}\right)\right\rangle .
$$

Let us determine first the terms $\sim(n F)^{0}$ :

$$
\frac{\lambda}{\tau n \varkappa^{2} T / m}=-\frac{\beta^{2}}{3} m\left\langle\frac{p^{2}}{2 m} \frac{p^{2}}{m^{2}}\left(\frac{5}{2}-\beta \frac{p^{2}}{2 m}\right)\right\rangle=\frac{5}{2} .
$$


This proves (24), the zero order thermal conductivity in the approximation (6). We are now interested in the virial corrections $\sim(n F)^{1}$ :

$$
\lambda=\frac{5}{2} \frac{\tau n \varkappa^{2} T}{m}\left(1+n \Delta_{\mathrm{s}}{ }^{2}\right) .
$$

Then it is

$$
\begin{aligned}
& \Delta_{\mathrm{s}}{ }^{\lambda}=-\frac{2}{15} m \beta^{2}\left[\frac{\mathrm{d}}{\mathrm{d} n}\left\langle\left(\varepsilon_{p} \frac{\partial \varepsilon_{p}}{\partial \boldsymbol{p}}+\beta n F(p, q) \varepsilon_{q} \frac{\partial \varepsilon_{q}}{\partial \boldsymbol{q}}\right) \cdot \frac{\partial \varepsilon_{p}}{\partial \boldsymbol{p}}\left(\frac{5}{2}+n B-n T B^{\prime}-\beta \varepsilon_{p}\right)\right\rangle\right]_{n=0}, \\
& \Delta_{\mathrm{s}}{ }^{\lambda}=-\frac{2}{15} m \beta^{2}\left\{\left(B-T B^{\prime}\right)\left\langle\frac{p^{2}}{2 m} \frac{p^{2}}{m^{2}}\right\rangle+\beta\left\langle F(p, q) \frac{q^{2}}{2 m} \frac{\boldsymbol{q} \cdot \boldsymbol{p}}{m^{2}}\left(\frac{5}{2}-\beta \frac{p^{2}}{2 m}\right)\right\rangle+\left[\frac{\mathrm{d}}{\mathrm{d} n}\left\langle\varepsilon_{p} \frac{\partial \varepsilon_{p}}{\partial \boldsymbol{p}} \cdot \frac{\partial \varepsilon_{p}}{\partial \boldsymbol{p}}\left(\frac{5}{2}-\beta \varepsilon_{p}\right)\right\rangle\right]_{n=0}\right\} .
\end{aligned}
$$

In the last term one has to watch that besides the $n$-dependence of $\varepsilon_{p}$ also the weight functions depend on the density.

$$
\begin{aligned}
\Delta_{\mathrm{s}}{ }^{2}=- & \left(B-T B^{\prime}\right)-\frac{2}{15} \beta^{2}\left\{\beta\left\langle F(p, q) \frac{q^{2}}{2 m} \frac{\boldsymbol{p} \cdot \boldsymbol{q}}{m}\left(\frac{5}{2}-\beta \frac{p^{2}}{2 m}\right)\right\rangle\right. \\
& +\left\langle F(p, \bar{q}) \frac{p^{2}}{m}\left(\frac{5}{2}-\beta \frac{p^{2}}{2 m}\right)\right\rangle+2\left\langle\frac{p^{2}}{2 m} \frac{\partial}{\partial \boldsymbol{p}} F(p, \bar{q}) \cdot \frac{\partial}{\partial \boldsymbol{p}} \frac{p^{2}}{2}\left(\frac{5}{2}-\beta \frac{p^{2}}{2 m}\right)\right\rangle \\
+\left\langle\frac{p^{2}}{2 m} \frac{p^{2}}{m}(-\beta) F(p, \bar{q})\right\rangle & \left.+\left\langle\frac{p^{2}}{2 m} \frac{p^{2}}{m}\left(\frac{5}{2}-\beta \frac{p^{2}}{2 m}\right)(-\beta) F(p, \bar{q})\right\rangle+\left\langle\frac{p^{2}}{2 m} \frac{p^{2}}{m}\left(\frac{5}{2}-\beta \frac{p^{2}}{2 m}\right)\right\rangle \beta\langle F\rangle\right\} .
\end{aligned}
$$

The last term is $2 B ; \partial F / \partial \boldsymbol{p}$ can be integrated by parts, again taking care of the distribution function.

$$
\begin{aligned}
-\frac{15}{4}\left(\Delta_{\mathrm{s}}{ }^{2}-B-T B^{\prime}\right)=\left\langle\frac { \beta } { 2 } F \left\{-2 \frac{\partial}{\partial \boldsymbol{p}} \cdot\right.\right. & {\left[\frac{\beta p^{2}}{2 m}\left(\frac{5}{2}-\frac{\beta p^{2}}{2 m}\right) \boldsymbol{p}\right]-2 \frac{\beta p^{2}}{2 m} \boldsymbol{p} \cdot\left(\frac{5}{2}-\frac{\beta p^{2}}{2 m}\right)\left(-\beta \frac{\boldsymbol{p}}{m}\right) } \\
& \left.\left.-\frac{\beta p^{2}}{2 m} \frac{\beta p^{2}}{m}+\left(\frac{5}{2}-\frac{\beta p^{2}}{2 m}\right)\left(\frac{\beta q^{2}}{2 m} \beta \frac{\boldsymbol{p} \cdot \boldsymbol{q}}{m}+\frac{p^{2}}{m}-\frac{\beta p^{2}}{2 m} \frac{p^{2}}{m}\right)\right\}\right\rangle .
\end{aligned}
$$

Using relative and total momentum, we have

$$
\begin{aligned}
& \frac{\beta K^{2}}{4 m} \equiv e_{K}, \quad \frac{\beta k^{2}}{m} \equiv e_{k}, \\
& \beta \frac{\boldsymbol{p} \cdot \boldsymbol{q}}{m}=e_{K}-e_{k}, \quad\left(\frac{\beta K \cdot k}{m}\right)^{2} \triangleq \frac{4}{3} e_{K} e_{k} .
\end{aligned}
$$

The last equality holds in the average over $K$ or $k$ resp. Odd powers of $\boldsymbol{K} \cdot \boldsymbol{k}$ vanish in the average. Using

$$
\left\langle e_{K}\right\rangle=\frac{3}{2}, \quad\left\langle e_{K}^{2}\right\rangle=\frac{15}{4}, \quad\left\langle e_{K}^{3}\right\rangle=\frac{105}{8}
$$

one finds after some straight forward calculations

$$
-15\left(\Delta_{\mathrm{s}}{ }^{2}-B-T B^{\prime}\right)=-\frac{45}{4} F_{0}+\frac{65}{2} F_{1}-F_{2}+2 F_{3} \text {. }
$$

Here $\quad F_{j}=\left\langle\frac{1}{2} \beta F(k)\left(\beta k^{2} / m\right)^{j}\right\rangle$

has been given in (7), (41), (42) for $j=0,1,2$. As $\boldsymbol{Q}$ is given by a higher moment of the velocity $\partial \varepsilon_{p} / \partial \boldsymbol{p}$ as $P_{v_{\mu}}$, a higher moment of $F(k)$, i. e. $B^{\prime \prime \prime}$ is needed for $\Delta_{\mathrm{s}}{ }^{\lambda}$.

One easily proves

$$
F_{3}=\frac{9}{2} F_{2}+T \frac{\partial}{\partial T} F_{2} .
$$

Rearrangement in (52) thus gives the final result $\Delta_{\mathrm{s}}{ }^{2}=-\frac{1}{15}\left(125 B+105 T B^{\prime}+26 T^{2} B^{\prime \prime}+2 T^{3} B^{\prime \prime \prime}\right)$.
Using the Lennard-Jones-virial coefficient as tabulated in ${ }^{11}$ we find

$$
\begin{array}{c|cccc}
\text { Gas } & \mathrm{A} & \mathrm{Ne} & \mathrm{N}_{2} & \mathrm{O}_{2} \\
\cline { 1 - 5 } \Delta_{\mathrm{s}}{ }^{\lambda} / B & 3.4 & -10 & 25 & 3.9
\end{array}
$$

As $B<0$ at room temperature (except $\mathrm{Ne}$ ), we have $\Delta_{\mathrm{s}}{ }^{\lambda}<0$. Thus again the density dependence of the streaming operator tends to diminish the collisional effect of $\mathrm{d} \lambda / \mathrm{d} n$. Experimental values of $\Delta \lambda / \lambda$ at room temperature may be estimated from a few tables or graphs ${ }^{7,11}$. They are some $6-7 \%$ per atm in contrast to $0.6-0.8 \%$ per atm for $\Delta \eta / \eta$. Thus $\delta_{\lambda} \approx 10$ times $\delta_{\eta}$ experimentally. Our simplification of $\partial_{c} f / \partial t$ gives the same $\Delta_{c}$ for viscosity as well as thermal conductivity. But the streaming part shows this factor 10 (more precise: $\approx 25$ ) in the order of magnitude of $\Delta_{\mathrm{s}}{ }^{\lambda} / \Delta_{\mathrm{s}}{ }^{\eta}$.

We thus conclude that the Boltzmann-Landauequation (5) seems to be able to describe the measured density dependence of the transport coefficients. To get results which are comparable to (at the moment only scarcely available) accurate measured data, one has to integrate more or less numerical the solution $f_{1}$ of equ. (11). 